\title{
Article \\ Investigation of PtSGT1 and PtSGT4 Function in Cellulose Biosynthesis in Populus tomentosa Using CRISPR/Cas9 Technology
}

\author{
Yinxuan Xue ${ }^{1,+}$, Siyan Li ${ }^{1,+}$, Deyu Miao ${ }^{1}$, Sai Huang ${ }^{1}$, Bin Guo ${ }^{1,2}$, Shanwen Li ${ }^{3}$ and Xin-Min An ${ }^{1, *}$ \\ 1 Beijing Advanced Innovation Center for Tree Breeding by Molecular Design, National Engineering \\ Laboratory for Tree Breeding, Key Laboratory of Genetics and Breeding in Forest Trees and Ornamental \\ Plants, Ministry of Education, College of Biological Sciences and Technology, Beijing Forestry University, \\ Beijing 100083, China; xueyinxuan@bjfu.edu.cn (Y.X.); kenneth1993@bjfu.edu.cn (S.L.); \\ miaodeyu@163.com (D.M.); HUANGSai@bjfu.edu.cn (S.H.); guobin531188058@163.com (B.G.) \\ 2 Institute of Forest Genetics and Tree Breeding, Shanxi Academy of Forestry and Grassland Sciences, \\ Taiyuan 030012, China \\ 3 Institute of Forest Culture, Shandong Academy of Forestry, Jinan 250014, China; lishanwen66@163.com \\ * Correspondence: anxinmin@bjfu.edu.cn \\ $\dagger$ Contributed equally to this work.
}

check for updates

Citation: Xue, Y.; Li, S.; Miao, D.; Huang, S.; Guo, B.; Li, S.; An, X.-M. Investigation of PtSGT1 and PtSGT4 Function in Cellulose Biosynthesis in Populus tomentosa Using

CRISPR/Cas9 Technology. Int. J. Mol. Sci. 2021, 22, 13200. https://doi.org/ $10.3390 /$ ijms222413200

Academic Editors: Matthias Fladung and Birgit Kersten

Received: 29 October 2021

Accepted: 2 December 2021

Published: 7 December 2021

Publisher's Note: MDPI stays neutral with regard to jurisdictional claims in published maps and institutional affiliations.

Copyright: (c) 2021 by the authors. Licensee MDPI, Basel, Switzerland. This article is an open access article distributed under the terms and conditions of the Creative Commons Attribution (CC BY) license (https:// creativecommons.org/licenses/by/ $4.0 /)$.

\begin{abstract}
Cellulose synthesis is a complex process in plant cells that is important for wood processing, pulping, and papermaking. Cellulose synthesis begins with the glycosylation of sitosterol by sitosterol glycosyltransferase (SGT) to produce sitosterol-glucoside (SG), which acts as the guiding primer for cellulose production. However, the biological functions of SGTs in Populus tomentosa (P. tomentosa) remain largely unknown. Two full-length PtSGT genes (PtSGT1 and PtSGT4) were previously isolated from $P$. tomentosa and characterized. In the present study, CRISPR/Cas9 gene-editing technology was used to construct PtSGT1-sgRNA and PtSGT4-sgRNA expression vectors, which were genetically transformed into P. tomentosa using the Agrobacterium-mediated method to obtain transgenic lines. Nucleic acid and amino acid sequencing analysis revealed both base insertions and deletions, in addition to reading frame shifts and early termination of translation in the transgenic lines. Sugar metabolism analysis indicated that sucrose and fructose were significantly downregulated in stems and leaves of mutant PtSGT1-1 and PtSGT4-1. Glucose levels did not change significantly in roots and stems of PtSGT1-1 mutants; however, glucose was significantly upregulated in stems and downregulated in leaves of the PtSGT4-1 mutants. Dissection of the plants revealed disordered and loosely arranged xylem cells in the PtSGT4-1 mutant, which were larger and thinner than those of the wild-type. This work will enhance our understanding of cellulose synthesis in the cell walls of woody plants.
\end{abstract}

Keywords: sitosterol glycosyltransferase; Populus tomentosa; gene editing; cellulose synthesis; cell wall

\section{Introduction}

Cell walls are important components of plant cells responsible for their characteristic structures. In addition to protecting and supporting plant cells, cell walls contribute to their physiological functions, such as material transport and signal transduction. Cellulose is the most important component of the plant cell wall. The basic unit of cellulose is pyranoid D-glucose, which is linked by $\beta-1,4$-glycosidic bonds. In plants, sterol biosynthesis occurs through the mevalonate and non-mevalonate pathways [1], which have highly diverse skeletal and glycosylated structural forms. Sterols and their derivatives are involved in the structure of cell membranes [2], and act as precursors for hormone and vitamin biosynthesis [3]. Other important cellular functions of sterols include anti-stress and antiheat shock actions, signal transduction, and protection of bacteria from the host immune response [4]. Some sterols, such as sitosterol, are ubiquitous in plants [5]. 
Glycosyltransferases are a multigene family of enzymes that catalyze the glycosylation of biomolecules; there are 98 families distinguished according to amino acid sequence similarity [6]. Sitosterol glycosyltransferase (SGT) catalyzes the glycosylation of phytosterols and is associated with membrane binding [7]. Sitosterol-glucoside (SG) is derived from sterol, a component of the plant cell membrane [8], and is used as a primer for cellulose synthesis [9]. Cellulose synthase (CesA) utilizes uridine $5^{\prime}$-diphosphoglucose (UDP-Glu) as a substrate to form glucan chains as $\beta-1,4-$ glycosidic bonds [9]. Peng [10] demonstrated that cotton fiber membranes synthesize sitosterol- $\beta$-glucoside (SG), while UDP-Glu synthesized sitosterol-cellulose dextrin (SCD) in the presence of pre-synthesized cellulose. As a primer, SG participated in the initial cellulose synthesis. Sucrose is one of the photosynthetic products transported from source to sink in plants [11]. Sucrose synthase (SUS, EC 2.4.1.13) is the major sucrolytic enzyme [12]. It catalyzes a reversible reaction, but preferentially converts sucrose into fructose and UDP-Glu in planta [12], while as the substrate of glucan chains polyreaction, UDP-Glu is involved in cellulose synthesis in the cell walls of woody plants.

The proposed function of the Korrigan (Kor) protein is cleavage of SG from SCD, which allows the chain to be more efficiently extended. Studies have shown that cellulose biosynthesis occurs in herbicide-treated cotton fibers co-transformed with SG and cellulose fragments, indicating the importance of these biomolecules as primers for cellulose biosynthesis in higher plants [10]. Schrick et al. [13] reported that the phytosterol biosynthetic mutants fackel, hydra1, and phytosterol methyltransferase reduced cellulose levels. Using UDP-Glu and $\beta$-sitosterol as substrates, Li et al. [14] found that GhSGT1 and GhSGT2 recombinant proteins in cotton displayed different SG enzyme activities. The biochemical properties of GhSGT2 are similar to those of GhCESAs, and GhSGT2 may play an important role in the cellulose biosynthesis of cotton fibers. Many reports on cellulose synthesis exist, with most being focused on the role of the SGT gene in cellulose synthesis in herbaceous plants, such as Arabidopsis thaliana [15] and cotton [14]; however, there are few studies on woody plants. Studying the SGT gene in $P$. tomentosa could provide a foundation for further analysis of cellulose synthesis in woody plants.

Poplar is a fast-growing tree with high economic and ecological value [16,17]. In 2006, the entire genome of Populus trichocarpa (P. trichocarpa) was published [18]. Many researchers have identified the poplar tree as a model system for tree biology due to its small genome, ease of cloning, and amenability to high-throughput transgenic techniques [19]. Liu et al. [20] analyzed the efficiency of mutagenesis by using single-guide RNA (sgRNA) targeting the phytoene desaturase (PtoPDS) DNA sequence of P. tomentosa. A mismatch was found between sgRNA and the target gene, leading to reduced efficiency of mutagenesis, and possible mutagenesis failure. Complementarity of sequences between the $3^{\prime}$-terminal nucleotides of sgRNA and target gene was found to be particularly important for effective mutagenesis. In another similar study, the method utilized CRISPR/Cas9 technology to destroy endogenous PtoPDS genes while also producing homologous and heterologous PDS mutants, indicating that the CRISPR/Cas9 system is an efficient and powerful tool for genomic modification of woody plants [21].

CRISPR/Cas9 technology was also used to knock out 4CL genes in poplar trees; $100 \%$ mutagenesis was achieved in two 4CL genes, supporting the specificity and effectiveness of the CRISPR/Cas9 system in these plants [22]. This provided a simple way to identify the functions of members of a polygenic family. Studies have shown that CRISPR/Cas9 technology effectively produces targeted mutations in transgenic poplars, and homozygous mutations are produced at the desired site in the first generation [21]. At present, most research on CRISPR/Cas9 technology focuses on model plants and crops, with few studies of woody plants. Effective mutation of woody plants by the CRISPR/Cas9 system will be beneficial by allowing for genetic enhancement of forest trees. In our previous preliminary analysis, four PtSGTs of eight total members were isolated from P. tomentosa and characterized (Supplementary Figure S1 and Supplementary Table S2). Combining with the characteristics of high expression of AtSGT1 and AtSGT4 in stems and leaves of 
Arabidopsis based on TAIR (TAIR — Home Page arabidopsis.org) database, of these, PtSGT1 and PtSGT4 were chosen for further functional identification due to their high expression in stems and leaves. In this study, the P. tomentosa PtSGT1 and PtSGT4 genes were mutated using CRISPR/Cas9, which altered the sugar content and xylem structure in the mutant lines. Our findings will further our understanding of cellulose biosynthesis in the cell walls of woody plants.

\section{Results}

\subsection{Cloning and Sequence Analysis of PtSGT1 and PtSGT4}

Following amplification with primers PtSGT1F, PtSGT1R, PtSGT4F, and PtSGT4R, a $1.9-\mathrm{kb}$ fragment was obtained by $1.5 \%$ agarose gel electrophoresis using $P$. tomentosa cDNA as the template (Figure 1). The sequencing results indicate that the full-length cDNAs of P. tomentosa PtSGT1 (GenBank acc. no. MG904686) and PtSGT4 (unpublished) are 1851 and $1935 \mathrm{bp}$, respectively, and the CDS sequences of both are complete open reading frames (ORF) encoding 616 and 644 amino acids, respectively.

TargetP 1.1 Server software was used to predict the subcellular localization of the two genes. PtSGT1 was thought to be located in the chloroplast, while PtSGT4 may be in the cytoplasm. Blast was used to analyze the nucleic acid sequence similarity of Populus trichocarpa, and indicated 94\% similarity between PtSGT1 and PtrSGT1 (Potri.014G178300.1), and 99\% similarity between PtSGT4 and PtrSGT4 (Potri.005G193100.1). The results of the BLASTP (protein-protein blast) comparison indicated that the amino acid sequences encoded by P. tomentosa PtSGT1 and PtSGT4 were $83 \%$ and $96 \%$ similar to those of P. trichocarpa, respectively.

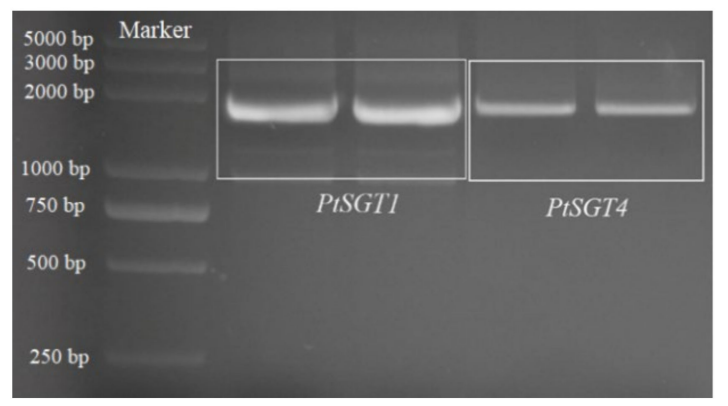

Figure 1. Electropherograms of PtSGT1 and PtSGT4 gene clones.

\subsection{Plasmid Construction}

Table 1 shows the sgRNAs designed for PtSGT1 and PtSGT4, respectively, which were integrated with the plasmid vector to obtain the recombinant expression vector VK00514 (Figure 2B). The positions of the sgRNAs in the genes are shown in Figure 2A. After being transferred into $E$. coli DH5 $\alpha$ cells, single-colony PCR detection was carried out, and positive E. coli colonies were collected for plasmid extraction. The tested vectors were introduced into Agrobacterium for verification by PCR. The PCR product was subjected to agarose electrophoresis, and positive clones were selected. The detected fragment size was consistent with the size of the target gene, indicating that the recombinant vector extracted from E. coli had been successfully transferred into Agrobacterium.

Table 1. The sgRNA target sequences.

\begin{tabular}{clc}
\hline Gene & Name of Plasmid & sgRNA Target Sequence (5' $\left.\rightarrow \mathbf{3}^{\prime}\right)$ \\
\hline PtSGT1 & VK005-14-PtSGT1 & CCCATACCTGTTGATGAGTTC \\
PtSGT4 & VK005-14-PtSGT4 & TAGATCCAAGACGGAGAACAGGG \\
\hline
\end{tabular}




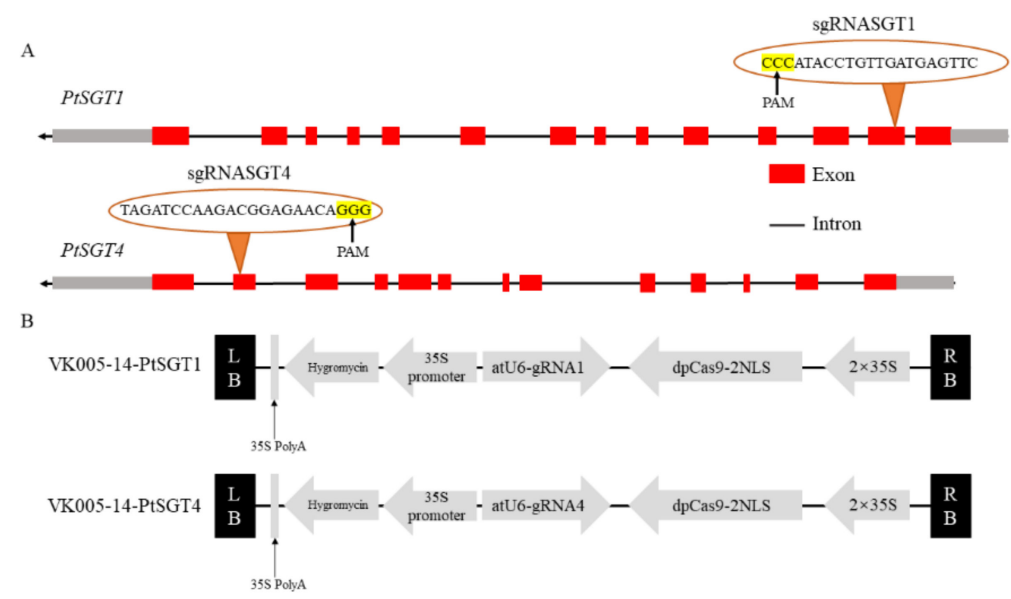

Figure 2. Design of the sgRNAs for the target genes and construction of the CRISPR/Cas9-sgRNA structure: (A) Target sequence selection site for the PtSGT genes. Untranslated regions (UTRs) are shown in gray boxes. (B) Schematic illustration of the CRISPR/Cas9-sgRNA structure (the backbone is VK005-14, dpCas9: SpCas9 optimized for dicotyledons, NLS: nuclear localization sequence).

\subsection{Genetic Transformation of P. tomentosa}

Transgenic plants were obtained by transforming vector 'p2×35S-dpCas9-atU6-gRNA35sh-Hyg' into Agrobacterium tumefaciens (Figure 3), and DNA from the leaf of 30-day-grown mutant was extracted. This extracted DNA was used as the template and the universal detection primer was used for detection (Table 2). A total of 51 strains of positive plants were obtained and WT P. tomentosa was used as the negative control (Figure 4).

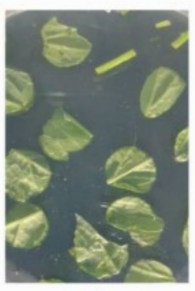

A

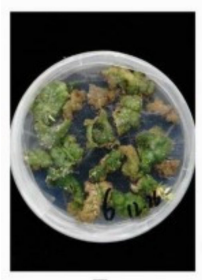

E

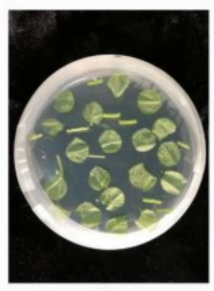

B

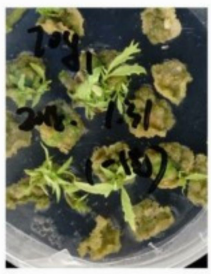

$\mathrm{F}$

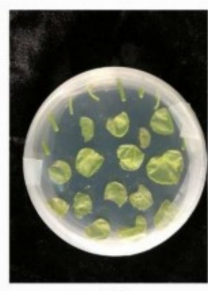

C

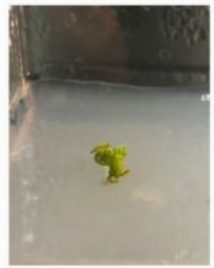

G

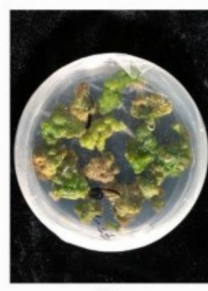

D

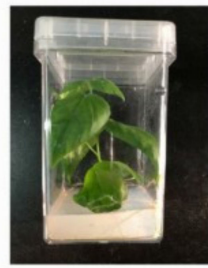

$\mathrm{H}$

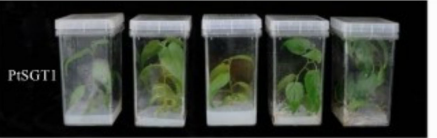

I

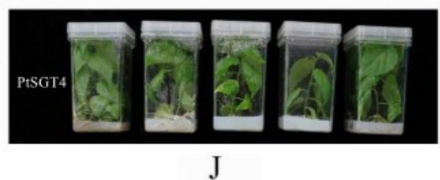

Figure 3. Agrobacterium-mediated genetic transformation of $P$. tomentosa: (A) plant tissue preculture (Murashige and Skoog (MS) medium with $2 \mathrm{mg} / \mathrm{L}$ 6-benzylaminopurine (6-BA) and $0.1 \mathrm{mg} / \mathrm{L}$ 1naphthylacetic acid (NAA)). (B,C) Culture after Agrobacterium infection (MS medium with $2 \mathrm{mg} / \mathrm{L}$ 6-BA and $0.1 \mathrm{mg} / \mathrm{L}$ NAA and $500 \mathrm{mg} / \mathrm{L}$ cefotaxime (Cef) and $1 \mathrm{mg} / \mathrm{L}$ hygromycin (Hyg)). (D-F) Adventitious bud growth (MS medium with $2 \mathrm{mg} / \mathrm{L}$ 6-BA and $0.1 \mathrm{mg} / \mathrm{L}$ NAA and $500 \mathrm{mg} / \mathrm{L}$ Cef and $1 \mathrm{mg} / \mathrm{L} \mathrm{Hyg}$ ). (G) Adventitious buds were transplanted into rooting medium (1/2 MS medium with $0.4 \mathrm{mg} / \mathrm{L}$ indole-3-butytric acid (IBA) and $500 \mathrm{mg} / \mathrm{L} \mathrm{Cef} \mathrm{and} 1 \mathrm{mg} / \mathrm{L} \mathrm{Hyg}$ ). (H) Adventitious buds developed into plants (1/2 MS medium with $0.4 \mathrm{mg} / \mathrm{L} \mathrm{IBA}$ and $500 \mathrm{mg} / \mathrm{L} \mathrm{Cef} \mathrm{and} 1 \mathrm{mg} / \mathrm{L}$ Hyg). (I,J) Plants to be tested (1/2 MS medium with $0.4 \mathrm{mg} / \mathrm{L} \mathrm{IBA})$. 


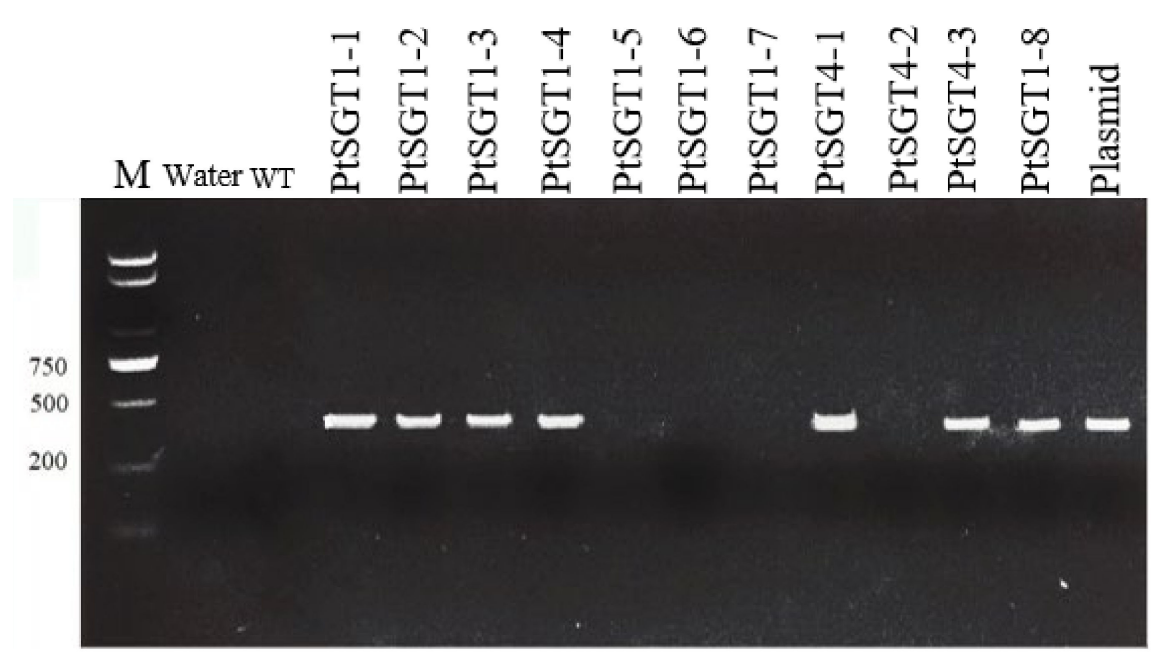

Figure 4. Part of the electrophoretic detection map of transgenic seedlings: M, marker (100-2000 bp, Sangon Biotech, Shanghai, China); Water, negative control; WT, wild-type; Plasmid, positive control. PtSGT1-1, 1-2, 1-3, 1-4, 1-6, 1-7, 1-8, and PtSGT4-1, 4-2, 4-3 represent different transformants, respectively.

Table 2. List of universal detection primers for plasmid vectors.

\begin{tabular}{cc}
\hline Primer & Sequence $\left.\mathbf{( 5}^{\prime} \rightarrow \mathbf{3}^{\prime}\right)$ \\
\hline Universal detection primer F & TCTTCAAAGTCCCACATCGC (Source: VK005-14) \\
Universal detection primer R & ACGCTAAGGGAATGCTTGTAT (Source: VK005-14) \\
\hline
\end{tabular}

Comparisons of WT and positive mutant sequences identified two target positive mutants of PtSGT1 (Figure 5) and PtSGT4 (Figure 6) with sequence changes. PtSGT1-2 and PtSGT1-3 had single base conversions, with the original G base changed to an A base, and a deletion of the G base at the same position of PtSGT1-1. The sequence of PtSGT4-1 had a single base increase. The amino acid of the WT PtSGT4 gene and transgenic types PtSGT1-1 and PtSGT4-1 products were compared using DNAMAN software, which identified large amino acid changes at the end of the PtSGT1 sequence (Figure 5). Few changes to the amino acid sequence of PtSGT4 were observed; however, the single base change in both resulted in the premature end of translation (Figure 6).

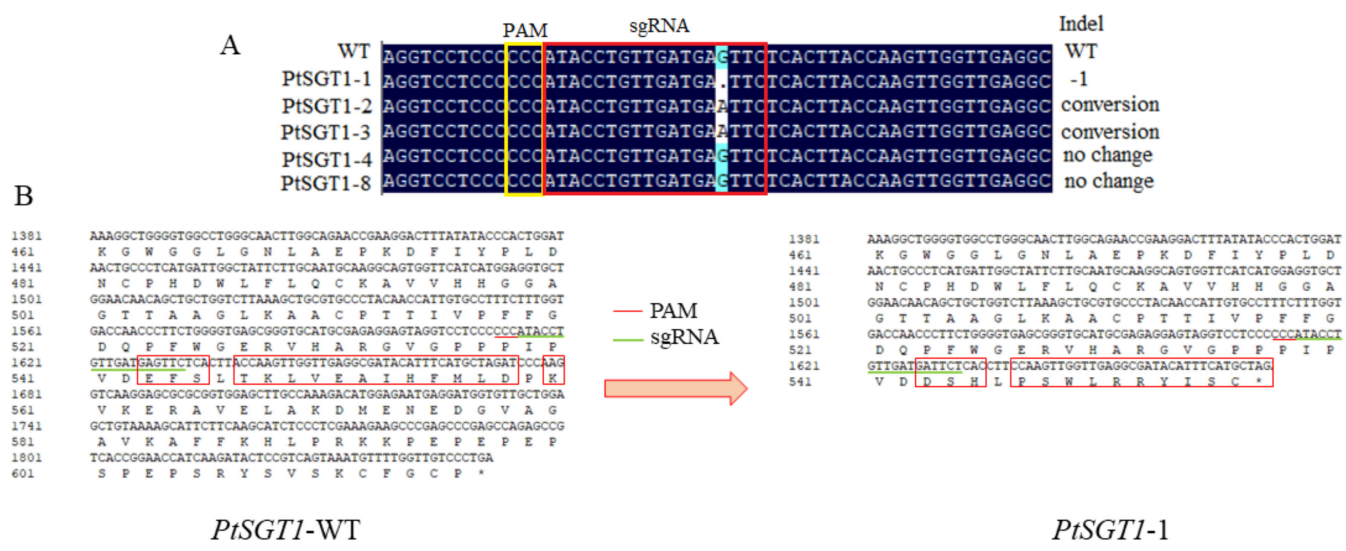

Figure 5. Mutation analysis of the target sequences in transgenic lines of P. tomentosa with edited PtSGT1: (A) results of the target sequencing, (B) comparison of the amino acid sequences of wild-type and a transgenic line with edited PtSGT1. 


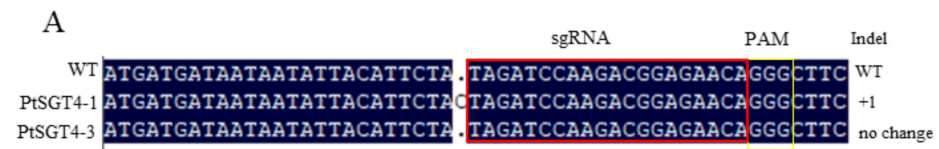

B

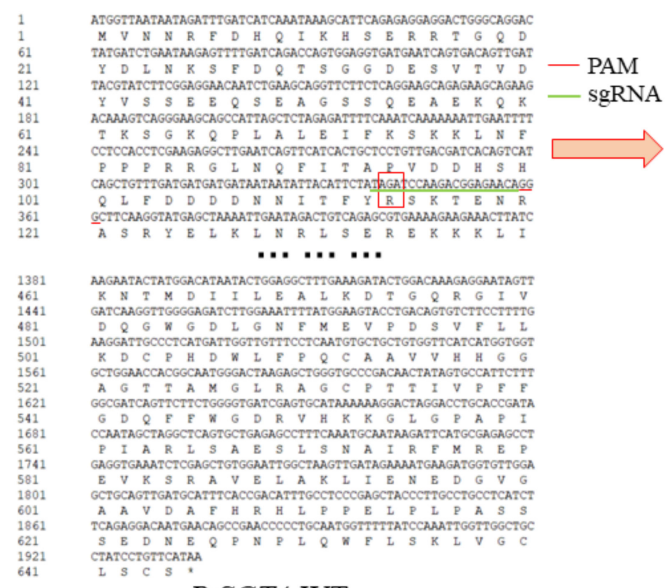

PtSGT4-WT

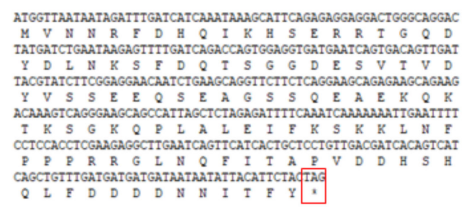

PtSGT4-1

Figure 6. Mutation analysis of the target sequences in transgenic lines of $P$. tomentosa with edited PtSGT4: (A) results of target sequencing. (B) Comparison of the amino acid sequences of wild-type and a transgenic line with edited PtSGT4.

\subsection{Sugar Extraction and Measurement}

Figure 7 shows the content of sucrose, fructose, and glucose in PtSGT1-1 and PtSGT4-1 transgenic plants. Sucrose and fructose levels in roots, stems, and leaves of the PtSGT1-1 mutant were significantly lower than those of WT P. tomentosa and gene-edited P. tomentosa, with the levels being reduced by $40-70 \%$. The glucose content in leaves of transgenic plants was significantly lower than in WT. Sucrose and fructose levels in roots, stems, and leaves of the PtSGT4-1 mutant were significantly lower than those of WT, while the glucose content in the stem was higher than that in WT.
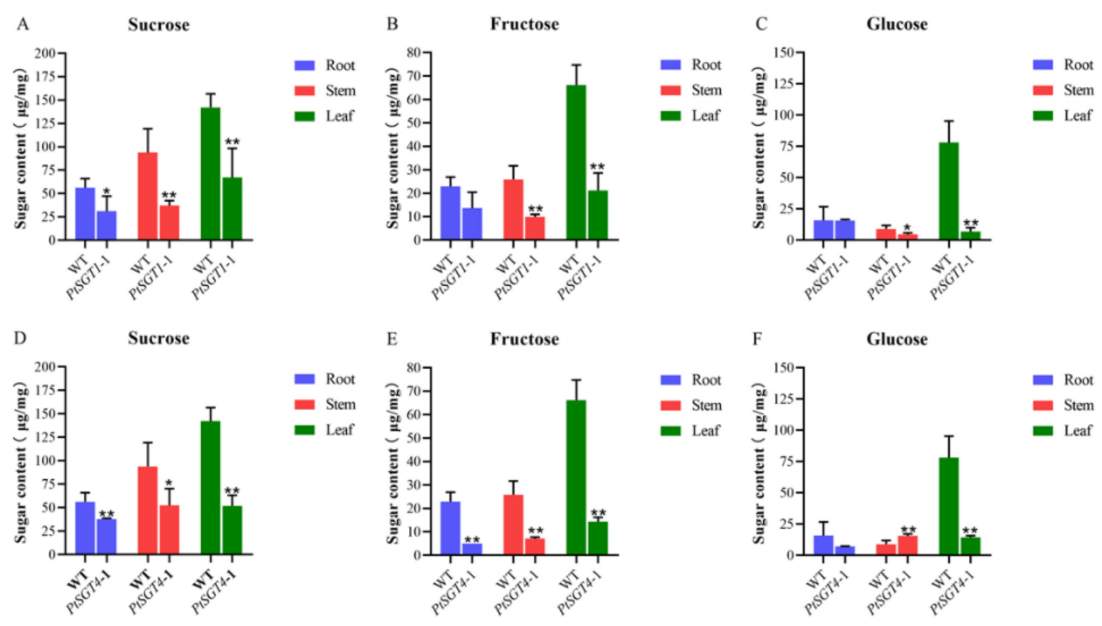

Figure 7. Comparison of sugar content between wild-type and transgenic type: (A-C) comparison of the sucrose, fructose, and glucose levels between the wild-type and transgenic PtSGT1-1; (D-F) comparison of the sucrose, fructose, and glucose levels between the wild-type and transgenic PtSGT4-1.

\subsection{Sections of Xylem from Transgenic Plants}

Wood from WT and mutant strains grown for 1 year was sliced by hand. Xylem sections from WT and mutant strains PtSGT1-1 and PtSGT4-1 were observed using the 
BX61 microscope (Olympus). Xylem cells from WT plants were arranged tightly and neatly, with small cells (Figure 8). Xylem cells from the mutants PtSGT1-1 and PtSGT4-1 were loosely arranged and were thinner and larger than those of WT cells. The parenchyma cells in the WT medulla were irregular and disordered in shape, while the parenchyma cells in transgenic PtSGT1-1 and PtSGT4-1 medulla were polygonal in shape and arranged in an orderly manner.
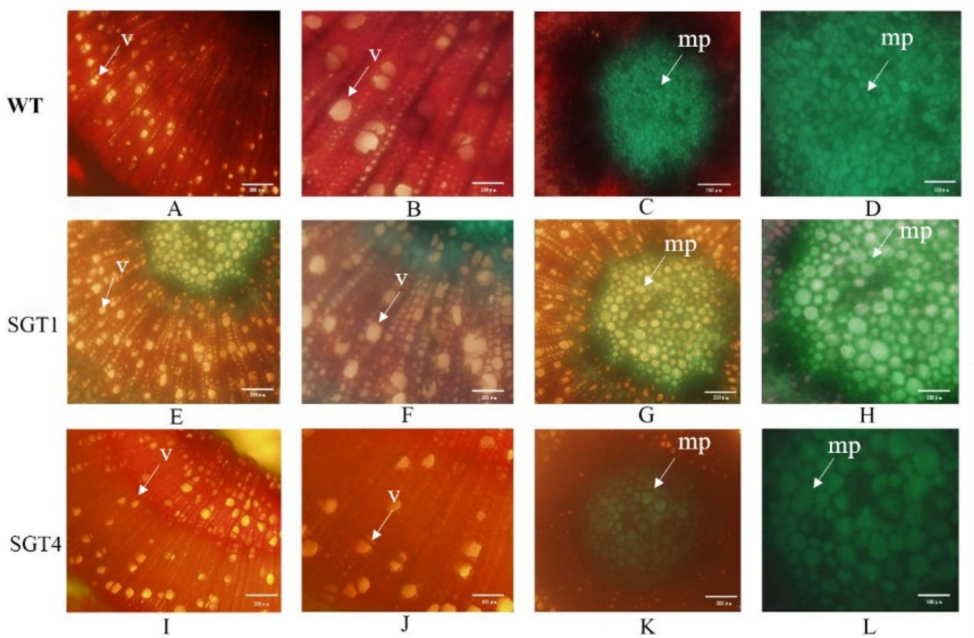

Figure 8. Xylem sections from wild-type and transgenic PtSGT1-1 and PtSGT4-1 plants. The arrows point to the vessel (v) and myeloid parenchyma (mp). Images (A-D) are $10 \times$ and $20 \times$ magnifications of xylem cells, and $10 \times$ and $20 \times$ magnifications of myeloid parenchyma cells from WT plants, respectively. Images $(\mathbf{E}-\mathbf{H})$ are $10 \times$ and $20 \times$ magnifications of xylem cells and $10 \times$ and $20 \times$ magnifications of medullary parenchyma cells from PtSGT1-1 plants, respectively. Images (I-L) are $10 \times$ and $20 \times$ magnification of xylem calls and $10 \times$ and $20 \times$ magnifications of myeloid parenchyma cells from PtSGT4-1 plants, respectively.

\section{Discussion}

Cellulose synthesis is a complex process in plant cells, while SGT plays an essential role in cellulose synthesis in the cell wall. A previous study found that SG synthesis occurs on the inner surface of the plasma membrane of plant cells and is involved in responses to phytohormones and the initiation reaction of the cellulose synthesis pathway [10]. The mutation of the Arabidopsis thaliana co-chaperone SGT1b impairs responses to the plant hormones jasmonate, auxin, and gibberellic acid, but not brassinolide and abscisic acid, and that $S G T 1 b$ and its homologue SGT1a are involved in maintaining the steady state levels of the F-box proteins COI1 and TIR1, receptors for jasmonate and auxin, respectively [15]. In cotton, two full-length GhSGT genes were identified, their distinct biochemical properties were also examined. Using UDP-Glu and $\beta$-sitosterol or total crude membrane sterols as substrates, GhSGT1 and GhSGT2 recombinant proteins were detected with different enzymatic activities for SG production [14]. These studies provided a basis for understanding the biological functions of $S G T$ gene in plants. In this study, we mutated the P. tomentosa SGT1 and SGT4 genes using the CRISPR/Cas9 system, and the results showed that sugar contents of the mutants were significantly different from those of the wild-type. The glucose content in the stem of the PtSGT1-1 mutant did not change significantly compared with that in wild-type. Mutation of the plant may have occurred at the $3^{\prime}$-end, which would not alter its function. Further microscopic observation showed that the arrangement and size of parenchyma cells in the xylem and pith of the mutant were altered. It can be inferred from the results that the SGT gene plays a role in the initial reaction of cellulose synthesis, and primarily acts in the xylem region of the stem. The results additionally indicate that SGT could affect the arrangement and size of xylem parenchyma cells. Microscopic observation of the xylem confirmed this result, and 
the cell wall of the mutant was different from that of wild-type. In addition, we found that the relative expression of PtSGT3 was greater than PtSGT1, PtSGT2, and PtSGT4 in poplar tissues, with the highest expression in stems in a previous study [23]. This suggests that PtSGT3 may play a crucial role in cellulose synthesis. Therefore, it will be listed as a priority target gene in our future research.

CRISPR/Cas9 has become a powerful tool for functional analysis of desired genes and been widely applied in many plants, including Arabidopsis, rice, potato, maize, and tobacco [24-31]. As CRISPR/Cas9 technology advances, researchers are turning their attention to woody plants. Liu et al. [20] assessed the efficiency of mutagenesis using different sgRNAs targeting the PDS gene of $P$. tomentosa and found that a mismatch between the sgRNA and target gene led to reduced efficiency of mutagenesis and the possibility of mutagenesis failure. Fan et al. [21] proposed an improved method for infusing Cas9-encoding genes and multiple sgRNAs into plant cells through a single plasmid. CRISPR/Cas9 technology was used in combination with this method to destroy endogenous PtoPDS genes in poplar trees. They successfully detected homologous and heterologous PDS mutants, showing that the CRISPR/Cas9 system is an efficient and powerful tool for genomic modification in woody plants. In this study, CRISPR/Cas9 was used to edit the P. tomentosa SGT gene. This produced insertion and deletion mutations in the mutant lines, which resulted in changes in the amino acid sequence, predicting that cellulose synthesis may be affected. The changes in glucose metabolism in mutants provided solid evidence. The glucose content in the stem of the PtSGT4-1 mutant was higher than in the control, which may be due to the large segment mutation at the $5^{\prime}$-end of the PtSGT4-1 plant, resulting in weakened or lost function of the SGT gene that could result in continuous accumulation of glucose in the substrate. Due to the high genetic diversity and complex genetic background of $P$. tomentosa, SNPs may exist between different genotypes. Therefore, designing multiple sgRNAs for a target gene and multi-gene editing would be a better strategy for increasing the mutation efficiency in our future work.

\section{Materials and Methods}

\subsection{Experimental Materials}

Tissue-cultured plantlets of $P$. tomentosa (TC1521, female clone) were used for DNA and RNA extraction and genetic transformation. Transformed materials were grown and maintained in the National Forestry Engineering Laboratory of Tree Breeding at Beijing Forestry University, Beijing, China. The plants were cultured in 1/2 Murashige and Skoog solid medium at $25{ }^{\circ} \mathrm{C}$, under $60 \%$ relative humidity and a $16 \mathrm{~h}$ light $/ 8 \mathrm{~h}$ dark photoperiod. The illuminance was 8000-10,000 lx. Agrobacterium tumefaciens GV3101 and Escherichia Coli (E. coli) Top10 were purchased from Tiangen Biotech (Beijing, China) [32].

\subsection{Cloning and Sequence Analysis of PtSGT}

Total RNA from selected plants was extracted using the SV Total RNA Isolation System kit (Promega, Madison, WI, USA), followed by removal of genomic DNA with RQ1 DNase. Finally, cDNA was synthesized using the Reverse Transcription System according to the manufacturer's instructions. Based on the CDS of PtrSGT1 and PtrSGT4, DNAMAN v6.0 software was used to design the primers for amplification of PtSGT1 and PtSGT4 (Table 3). TargetP 1.1 Server software was used to predict the subcellular localization of the two genes. The PCR reaction amplification system $(20 \mu \mathrm{L})$ included $10 \times$ PCR Buffer $(2.0 \mu \mathrm{L}), 2 \mathrm{mM}$ dNTPs $(1.6 \mu \mathrm{L})$, forward primers $(0.4 \mu \mathrm{L})$, reverse primers $(0.4 \mu \mathrm{L})$, cDNA template $(2.0 \mu \mathrm{L})$, Taq DNA polymerase $(0.2 \mu \mathrm{L})$, and $\mathrm{ddH}_{2} \mathrm{O}(13.4 \mu \mathrm{L})$. The PCR cycle conditions were as follows: $94{ }^{\circ} \mathrm{C}$ for $3 \mathrm{~min}$ for pre-denaturation, denaturation at $94{ }^{\circ} \mathrm{C}$ for $30 \mathrm{~s}$, annealing at $55^{\circ} \mathrm{C}$ for $30 \mathrm{~s}$, and extension at $72{ }^{\circ} \mathrm{C}$ for $90 \mathrm{~s}$. After 34 cycles, the reactions were extended for $7 \mathrm{~min}$ at $72{ }^{\circ} \mathrm{C}$ before being preserved at $4{ }^{\circ} \mathrm{C}$. 
Table 3. PCR primer sequences for PtSGT1 and PtSGT4 amplification and mutation sequencing analysis.

\begin{tabular}{ccc}
\hline Primer & Sequence $\left(\mathbf{5}^{\prime} \rightarrow \mathbf{3}^{\prime}\right)$ & Purpose \\
\hline PtSGT1F & ATGGCGGAGTCGCAGC & \\
PtSGT1R & TCAGGGACAACCAAAACATTTACT & Used for PtSGT1 and PtSGT4 amplification \\
$P t S G T 4 F$ & ATGGTTAATAATAGATTTGATCATC & \\
$P t S G T 4 R$ & TTATGAACAGGATAGGCAG & Used for mutation sequencing analysis \\
$P t S G T 1 F t$ & TATGCTTGTAATACCGATGC & \\
$P t S G T 1 R t$ & TGAGTAGCCAGTCTAACACGAT & \\
$P t S G T 4 F t$ & CAGAAGACAAAGTCAGGGAA & \\
\hline$t S G T 4 R t$ & TCTAACAACTCCGAAGCAAC & \\
\hline
\end{tabular}

\subsection{Construction and Transformation of the PtSGT Vector and Molecular Identification of Transformed P. tomentosa}

Using the exon sequence of the SGT gene, PtSGT1-sgRNA and PtSGT4-sgRNA were designed using the online tool CRISPOR (http:/ / crispor.tefor.net/, accessed on 29 October 2021) and assembled into a VK005-14 vector (Beijing Viewsolid Biotechnology Co. Ltd., Beijing, China). The target sgRNA design followed basic principles: the length of the sgRNA target sequence is generally about $20 \mathrm{bp}$; the $3^{\prime}$ end of the sgRNA target sequence contains GG, and due to its relative specificity, ending with more than 4 T nucleotides is avoided, and the GC content is $40-60 \%$; the number of base matches between the sgRNA sequence and off-target sites should be as small as possible; the PAM (protospacer adjacent motif) NGG motif should be included in the design of the sgRNA target location, but the PAM region should not be included in the design of carrier primers. The integrated vector plasmid VK005-14 was transferred into E. coli for single colony PCR detection. After successful detection, positive $E$. coli liquid was extracted for plasmid extraction. The plasmid verified by PCR was introduced into Agrobacterium tumefaciens by freeze-thaw method. Explants ( 2 months old) were obtained from aseptic tissue culture seedlings of the P. tomentosa female strain TC1521. Explants were transformed using Agrobacterium-mediated leaf disc transformation, with wild-type (WT) plants as controls. The transformation of P. tomentosa was performed as described in our previous study [32]. Genomic DNA was extracted from the transformed and WT plants using the Plant Genomic DNA Extraction Kit (Tiangen Biotech, Beijing, China) and served as the PCR template. PCR was performed at $94{ }^{\circ} \mathrm{C}$ for $5 \mathrm{~min}$, followed by 34 cycles of amplification $\left(94^{\circ} \mathrm{C}\right.$ for $30 \mathrm{~s}, 58^{\circ} \mathrm{C}$ for $30 \mathrm{~s}$, and $72{ }^{\circ} \mathrm{C}$ for $1 \mathrm{~min}$ ), with a final extension at $72^{\circ} \mathrm{C}$ for $5 \mathrm{~min}$. The PCR products were analyzed using $1.5 \%$ agarose gel electrophoresis [32]. Primers used for mutation sequencing analysis are listed in Table 1. The thermal cycles followed the same conditions mentioned above.

\subsection{Sugar Concentration Measurements in Transgenic Line Tissues}

The root, stem, and leaf tissues of mutant and wild-type (TC1521) P. tomentosa were collected. Three duplicate samples were taken from each tissue. Sugars were extracted by drying fresh tissue in an oven and grinding the sample. Samples $(\sim 10 \mathrm{mg})$ were added to $0.8 \mathrm{~mL}$ of $80 \%$ ethanol solution, placed in a water bath for $40 \mathrm{~min}$ at $80{ }^{\circ} \mathrm{C}$, and centrifuged $(10,000 \mathrm{rpm}, 1 \mathrm{~min})$ before collecting the supernatant. These steps were repeated before the addition of $\sim 2 \mathrm{mg}$ activated carbon to the supernatant. The supernatant was decolorized for $30 \mathrm{~min}$ at $80^{\circ} \mathrm{C}$ and centrifuged $(10,000 \mathrm{rpm}, 1 \mathrm{~min})$ before adding $80 \%$ ethanol solution for a final volume of $2 \mathrm{~mL}$ [32].

Sucrose content was measured in the transgenic lines by adding $\mathrm{NaOH}(0.1 \mathrm{~mL}$; $2 \mathrm{~mol} / \mathrm{L})$ to the sugar extract $(0.2 \mathrm{~mL})$ and boiling the reaction mixture in water for $5 \mathrm{~min}$. HCL $(1.4 \mathrm{~mL} ; 30 \%)$ and resorcinol $(0.4 \mathrm{~mL} ; 0.1 \%)$ were added to the reaction and the mixture was shaken at $80{ }^{\circ} \mathrm{C}$ for $5 \mathrm{~min}$. The absorbance of the reaction was measured at $480 \mathrm{~nm}$ to determine the sugar concentration. Fructose content was analyzed by adding $\mathrm{HCl}(0.8 \mathrm{~mL} ; 30 \%)$ and resorcinol $(0.8 \mathrm{~mL} ; 0.1 \%)$ to a $2 \mathrm{~mL}$ tube containing $0.4 \mathrm{~mL}$ of sugar extract. Following incubation at $80{ }^{\circ} \mathrm{C}$ for $10 \mathrm{~min}$, the absorbance was measured at $520 \mathrm{~nm}$ to determine the fructose concentration. The glucose content was analyzed by incubating 
$100 \mathrm{~mL}$ enzyme preparations (10 mg horseradish peroxidase, $10 \mathrm{mg}$ o-dianisidine- $\mathrm{HCl}$, $0.1 \mathrm{~mL}$ glucose oxidase (dissolved with $1 \mathrm{mg} \mathrm{50,000} \mathrm{U}$ glucose peroxidase in $0.1 \mathrm{~mol} / \mathrm{L}$ glacial acetic acid, pH 5.5) in a constant volume to $100 \mathrm{~mL}$ ) at $30^{\circ} \mathrm{C}$ for $5 \mathrm{~min}$, then $0.5 \mathrm{~mL}$ was added to $0.25 \mathrm{~mL}$ sugar extract, the mixture was shaken, and we then allowed it to sit for $5 \mathrm{~min}$. Finally, $\mathrm{H}_{2} \mathrm{SO}_{4}(1 \mathrm{~mL} ; 10 \mathrm{~mol} / \mathrm{L})$ was added to stop the reaction. The glucose concentration was determined by measuring the absorbance of the reaction mixture at $520 \mathrm{~nm}$. The calculation methods followed were the same as those seen in a previous publication [32].

\subsection{Sections of Xylem from Transgenic Plants}

Wood from WT and mutant strains grown for 1 year was sliced by hand. Xylem sections of WT and mutant strains PtSGT1-1 and PtSGT4-1 were observed and photographs were taken using a BX61 microscope (Olympus, Tokyo, Japan). The sections were placed in FAA (Formaldehyde-acetic acid-ethanol) solution ( $38 \%$ formaldehyde $5 \mathrm{~mL}$, glacial acetic acid $5 \mathrm{~mL}, 70 \%$ alcohol $90 \mathrm{~mL}$ ) for fixation for 15-60 min. The slices were transferred to a small Petri dish and dehydrated with 50,60, and 70\% ethanol for 5 min each. Then they were dyed for at least $30 \mathrm{~min}$ in $1 \%$ safranin dissolved in $70 \%$ ethanol. The stained slices were dehydrated with 70, 80, 90, and $95 \%$ ethanol for 5 min each. The sections were stained with $95 \%$ ethanol containing $1 \%$ fast green for $30 \mathrm{~s}$. The slices were placed in $95 \%$ ethanol for color separation and dehydrated with anhydrous ethanol for $5 \mathrm{~min}$. The sections were transferred to a 1:1 anhydrous ethanol: xylene mixture for $4 \mathrm{~min}$, and then transferred into pure xylene for $5 \mathrm{~min}$ to make the tissue sections transparent. Then, the section was placed on a slide with a drop of water, the cover slide was gently lowered, and the excess water was absorbed with filter paper, and then observed with a BX61 microscope.

\section{Conclusions}

In this study, we produced mutated P. tomentosa lines of PtSGT1 and PtSGT4 using CRISPR/Cas9. The results showed that there were some changes in sugar content and xylem structure in transgenic plants compared with wild-type. It is speculated that sucrose metabolism pathway is affected, suggesting important roles for PtSGT1 and PtSGT4 in cellulose synthesis. This work promotes our understanding of cellulose synthesis in the cell walls of woody plants.

Supplementary Materials: The following are available online at https:/ /www.mdpi.com/article/10 .3390/ijms222413200/s1.

Author Contributions: X.-M.A. designed and managed the project. Y.X. and S.L. (Siyan Li) completed experiments, B.G. and S.L. (Shanwen Li) collected materials, D.M. and S.H. participated in DNA extraction and PCR analysis. Y.X., S.L. (Siyan Li) and X.-M.A. wrote the manuscript. All authors approved the manuscript before submission. All authors have read and agreed to the published version of the manuscript.

Funding: This research was funded by the China National Key R\&D Program during the 14th Five-year Plan Period "Forest Tree Genome Editing Technology", and the National Natural Science Foundation of China (No. 31570661).

Institutional Review Board Statement: Not applicable.

Informed Consent Statement: Not applicable.

Data Availability Statement: The data presented in this study are openly available in the Sequence Read Archive of NCBI, the accession number of PtSGT1 is MG904686, and the accession number of PtSGT3 is MG904687. The remaining data are provided in the Supplementary Files.

Acknowledgments: All of experiments in this study had been completed in National Engineering Laboratory for Tree Breeding, College of Biological Sciences and Technology, Beijing Forestry University.

Conflicts of Interest: The authors have declared no conflicting or competing interests. 


\section{References}

1. Rohmer, M. The discovery of a mevalonate-independent pathway for isoprenoid biosynthesis in bacteria, algae and higher plants. Nat. Prod. Rep. 1999, 16, 565-574. [CrossRef]

2. Rog, T.; Pasenkiewicz-Gierula, M.; Vattulainen, I.; Karttunen, M. Ordering effects of cholesterol and its analogues. Biochim. Biophys. Acta 2009, 1788, 97-121. [CrossRef] [PubMed]

3. Khelashvili, G.; Rappolt, M.; Chiu, S.; Pabst, G.; Harries, D. Impact of Sterol Tilt on Membrane Bending Rigidity in Cholesterol and 7DHC-Containing DMPC Membrane. Biophys. J. 2012, 1021, 413a. [CrossRef]

4. Grille, S.; Zaslawski, A.; Thiele, S.; Plat, J.; Warnecke, D. The functions of steryl glycosides come to those who wait: Recent advances in plants, fungi, bacteria and animals. Prog. Lipid Res. 2010, 49, 262-288. [CrossRef] [PubMed]

5. Sharma, L.K.; Madina, B.R.; Chaturvedi, P.; Sangwan, R.S.; Tuli, R. Molecular cloning and characterization of one member of 3beta-hydroxy sterol glucosyltransferase gene family in Withania somnifera. Arch. Biochem. Biophys. 2007, 460, 48-55. [CrossRef]

6. Mackenzie, P.I.; Owens, I.S.; Burchell, B.; Bock, K.W.; Bairoch, A.; Belanger, A.; Fournel-Gigleux, S.; Green, M.; Hum, D.W.; Iyanagi, T.; et al. The UDP glycosyltransferase gene superfamily: Recommended nomenclature update based on evolutionary divergence. Pharmacogenetics 1997, 7, 255-269. [CrossRef] [PubMed]

7. Ullmann, P.; Ury, A.; Rimmele, D.; Benveniste, P.; Bouvier-Nave, P. UDP-glucose sterol beta-D-glucosyltransferase, a plasma membrane-bound enzyme of plants: Enzymatic properties and lipid dependence. Biochimie 1993, 75, 713-723. [CrossRef]

8. Warnecke, D.C.; Heinz, E. Purification of a Membrane-Bound UDP-Glucose: Sterol [beta]-D-Glucosyltransferase Based on Its Solubility in Diethyl Ether. Plant Physiol. 1994, 105, 1067-1073. [CrossRef] [PubMed]

9. Warnecke, D.C.; Baltrusch, M.; Buck, F.; Wolter, F.P.; Heinz, E. UDP-glucose:sterol glucosyltransferase: Cloning and functional expression in Escherichia coli. Plant Mol. Biol. 1997, 35, 597-603. [CrossRef] [PubMed]

10. Peng, L.; Kawagoe, Y.; Hogan, P.; Delmer, D. Sitosterol-beta-glucoside as primer for cellulose synthesis in plants. Science 2002, 295, 147-150. [CrossRef] [PubMed]

11. Goren, S.; Huber, S.C.; Granot, D. Comparison of a novel tomato sucrose synthase, SISUS4, with previously described SISUS isoforms reveals distinct sequence features and differential expression patterns in association with stem maturation. Planta 2011, 233, 1011-1023. [CrossRef] [PubMed]

12. Ruan, Y.L.; Llewellyn, D.J.; Furbank, R.T. Suppression of sucrose synthase gene expression represses cotton fiber cell initiation, elongation, and seed development. Plant Cell 2003, 15, 952-964. [CrossRef]

13. Schrick, K.; Fujioka, S.; Takatsuto, S.; Stierhof, Y.D.; Stransky, H.; Yoshida, S.; Jurgens, G. A link between sterol biosynthesis, the cell wall, and cellulose in Arabidopsis. Plant J. 2004, 38, 227-243. [CrossRef]

14. Li, X.; Xia, T.; Huang, J.; Guo, K.; Liu, X.; Chen, T.; Xu, W.; Wang, X.; Feng, S.; Peng, L. Distinct biochemical activities and heat shock responses of two UDP-glucose sterol glucosyltransferases in cotton. Plant Sci. 2014, 219, 1-8. [CrossRef] [PubMed]

15. Zhang, X.C.; Millet, Y.A.; Cheng, Z.; Bush, J.; Ausubel, F.M. Jasmonate signalling in Arabidopsis involves SGT1b-HSP70-HSP90 chaperone complexes. Nat. Plants 2015, 1, 15049. [CrossRef] [PubMed]

16. Choi, Y.I.; Noh, E.W.; Kim, H.J.; Shim, D. Overexpression of poplar GSTU51 confers selective tolerance to both mercury and methyl viologen but not to CDNB or cadmium in transgenic poplars. Plant Biotechnol. Rep. 2013, 7, 175-184. [CrossRef]

17. Sannigrahi, P.; Ragauskas, A.J.; Tuskan, G.A.; Oak Ridge National Lab. ORNL, O.R.T.U. Poplar as a feedstock for biofuels: A review of compositional characteristics. Biofuels Bioprod. Biorefin. 2010, 4, 209-226. [CrossRef]

18. Tuskan, G.A.; DiFazio, S.; Jansson, S.; Bohlmann, J.; Grigoriev, I.; Hellsten, U.; Putnam, N.; Ralph, S.; Rombauts, S.; Salamov, A.; et al. The genome of black cottonwood, Populus trichocarpa (Torr. \& Gray). Science 2006, 313, 1596-1604.

19. Park, S.; Oh, S.; Han, K.H. Large-scale computational analysis of poplar ESTs reveals the repertoire and unique features of expressed genes in the poplar genome. Mol. Breed. 2004, 14, 429-440. [CrossRef]

20. Liu, T.; Fan, D.; Ran, L.; Jiang, Y.; Liu, R.; Luo, K. Highly efficient CRISPR/Cas9-mediated targeted mutagenesis of multiple genes in Populus. Hereditas 2015, 37, 1044-1052. [PubMed]

21. Fan, D.; Liu, T.; Li, C.; Jiao, B.; Li, S.; Hou, Y.; Luo, K. Efficient CRISPR/Cas9-mediated Targeted Mutagenesis in Populus in the First Generation. Sci. Rep. 2015, 5, 12217. [CrossRef] [PubMed]

22. Zhou, X.; Jacobs, T.B.; Xue, L.; Harding, S.A.; Tsai, C. Exploiting SNPs for biallelic CRISPR mutations in the outcrossing woody perennial Populus reveals 4-coumarate: CoA ligase specificity and redundancy. New Phytol. 2015, 208, 298-301. [CrossRef]

23. Yan, L.S.; Bin, G.; An, X. Cloning and Expression Analysis of Two Sitosterol Glycosyltransferase Genes in Populus tomentosa. Chin. J. Cell Biol. 2018, 40, 1130-1137.

24. Jiang, W.; Zhou, H.; Bi, H.; Fromm, M.; Yang, B.; Weeks, D.P. Demonstration of CRISPR/Cas9/sgRNA-mediated targeted gene modification in Arabidopsis, tobacco, sorghum and rice. Nucleic Acids Res. 2013, 41, e188. [CrossRef]

25. Gao, Y.; Zhang, Y.; Zhang, D.; Dai, X.; Estelle, M.; Zhao, Y. Auxin binding protein 1 (ABP1) is not required for either auxin signaling or Arabidopsis development. Proc. Natl. Acad. Sci. USA 2015, 112, 2275-2280. [CrossRef] [PubMed]

26. Lowder, L.G.; Zhang, D.; Baltes, N.J.; Paul, J.R.; Tang, X.; Zheng, X.; Voytas, D.F.; Hsieh, T.F.; Zhang, Y.; Qi, Y. A CRISPR/Cas9 Toolbox for Multiplexed Plant Genome Editing and Transcriptional Regulation. Plant Physiol. 2015, 169, 971-985. [CrossRef] [PubMed]

27. Schuster, M.; Schweizer, G.; Reissmann, S.; Kahmann, R. Genome editing in Ustilago maydis using the CRISPR-Cas system. Fungal Genet. Biol. 2016, 89, 3-9. [CrossRef] 
28. Andersson, M.; Turesson, H.; Nicolia, A.; Falt, A.; Samuelsson, M.; Hofvander, P. Efficient targeted multiallelic mutagenesis in tetraploid potato (Solanum tuberosum) by transient CRISPR-Cas9 expression in protoplasts. Plant Cell Rep. 2017, 36, 117-128. [CrossRef] [PubMed]

29. Chilcoat, D.; Liu, Z.B.; Sander, J. Use of CRISPR/Cas9 for Crop Improvement in Maize and Soybean. Prog. Mol. Biol. Transl. Sci. 2017, 149, 27-46.

30. Meng, X.; Hu, X.; Liu, Q.; Song, X.; Gao, C.; Li, J.; Wang, K. Robust genome editing of CRISPR-Cas9 at NAG PAMs in rice. Sci. China Life Sci. 2018, 61, 122-125. [CrossRef] [PubMed]

31. Svitashev, S.; Young, J.K.; Schwartz, C.; Gao, H.; Falco, S.C.; Cigan, A.M. Targeted Mutagenesis, Precise Gene Editing, and Site-Specific Gene Insertion in Maize Using Cas9 and Guide RNA. Plant Physiol. 2015, 169, 931-945. [CrossRef] [PubMed]

32. Li, J.; Gao, K.; Lei, B.; Zhou, J.; Guo, T.; An, X. Altered sucrose metabolism and plant growth in transgenic Populus tomentosa with altered sucrose synthase PtSS3. Transgenic Res. 2020, 29, 125-134. [CrossRef] [PubMed] 